\title{
Role of reaction kinetics and mass transport in glucose sensing with nanopillar array electrodes Venkataramani Anandan ${ }^{1}$, Xiaoling Yang1, Euihyeon Kim², Yeswanth L Rao ${ }^{1}$ and Guigen Zhang*1,3,4
}

\author{
Address: ${ }^{1}$ Micro/Nano Bioengineering Lab, Department of Biological and Agricultural Engineering, The University of Georgia, Athens, GA 30602 , \\ USA, ${ }^{2}$ Department of Biomedical Engineering, Georgia Tech and Emory University, Atlanta, GA 30332-0535, USA, ${ }^{3}$ Nanoscale Science and \\ Engineering Center, The University of Georgia, Athens, GA 30602, USA and ${ }^{4}$ Faculty of Engineering, The University of Georgia, Athens, GA 30602, \\ USA \\ Email: Venkataramani Anandan - ramani@uga.edu; Xiaoling Yang - yangxl@engr.uga.edu; Euihyeon Kim - ian.kim@gatech.edu; \\ Yeswanth L Rao - yeshy@uga.edu; Guigen Zhang* - gzhang@engr.uga.edu \\ * Corresponding author
}

Published: 10 October 2007

Journal of Biological Engineering 2007, I:5 doi:10.1 186/1754-1611-1-5

This article is available from: http://www.jbioleng.org/content/I/I/5

(C) 2007 Anandan et al; licensee BioMed Central Ltd.

This is an Open Access article distributed under the terms of the Creative Commons Attribution License (http://creativecommons.org/licenses/by/2.0), which permits unrestricted use, distribution, and reproduction in any medium, provided the original work is properly cited.
Received: 12 September 2007

Accepted: 10 October 2007

\begin{abstract}
The use of nanopillar array electrodes (NAEs) for biosensor applications was explored using a combined experimental and simulation approach to characterize the role of reaction kinetics and mass transport in glucose detection with NAEs. Thin gold electrodes with arrays of vertically standing gold nanopillars were fabricated and their amperometric current responses were measured under bare and functionalized conditions. Results show that the sensing performances of both the bare and functionalized NAEs were affected not only by the presence and variation of the nanoscale structures on the electrodes but also by the reaction kinetics and mass transport of the analyte species involved. These results will shed new light for enhancing the performance of nanostructure based biosensors.
\end{abstract}

\section{Background}

Biosensors are important devices for monitoring biological species in various processes of environmental, fermentation, food and medical concerns. The main challenges biosensors face include low sensitivity, poor specificity and proneness to fouling. The advent of nanotechnology presents some promising solutions for alleviating these problems. While many efforts have been devoted to improving the performances of biosensors by taking advantage of nanostructures or macrostructures with nanoscale features, the effort to elucidate the underlying mechanism governing the performances of biosensors enhanced with nanostructures is still scant.
This study intends to investigate the role of reaction kinetics and mass transport in biosensing when electrodes with nanoscale features are used. For this purpose, we used glucose biosensor as a model system. In a typical glucose biosensor, an enzyme such as glucose oxidase is immobilized onto the electrode surface $[1,2]$. The performance of such functionalized electrodes can be improved by either adjusting the spatial distribution of the enzyme or by modifying the morphology of the electrode surface. To achieve a high efficiency in immobilizing an enzyme onto the electrode surface, various techniques have been developed, such as the use of self-assembled monolayer [1-4], conducting polymers [5,6] and sol-gels [7]. Among these 
methods, the self-assembled monolayer (SAM) approach offers a better control for enzyme distribution at the molecular level, a high degree of reproducibility in enzyme immobilization and a short distance between the immobilized enzyme and the electrode surface $[1,4]$. The SAM approach, however, is limited by the amount of the enzyme that can be immobilized onto the electrode surface, which in turn will affect the sensing performance of the biosensor [8]. To increase the amount of immobilized enzyme various nanostructures such as nanotubes, nanoparticles and nanorods have been explored in order to increase the active surface area of the electrodes. For example, nanostructures like gold nanotubes [8], carbon nanotubes [5,9] and gold nanoparticles [10] have been incorporated into electrode surfaces and they exhibited better performance than conventional flat electrodes.

Recently Wang et al. [11] used nanostructured platinum electrodes functionalized with glucose oxidase for glucose detection. These electrodes showed a significant (two orders of magnitude) increase in glucose detection sensitivity as compared with a flat electrode, but the response of these electrodes to $\mathrm{K}_{4} \mathrm{Fe}(\mathrm{CN})_{6}$ was just 2.3 times that of the flat electrode. They attributed such sensitivity enhancements for glucose detection to the increased enzyme loading and improved retention of hydrogen peroxide near the electrode surface without examining systematically the role of reaction kinetics and mass transport. We believe that the electrical current response of these nanostructured electrodes is controlled by reaction kinetics, mass transport and the geometric topography of the nanostructures. Thus, to be able to understand the mechanism governing such an electrochemical process for the purpose of improving the performance of nanostructure based electrodes, it is necessary to investigate the role of reaction kinetics and mass transport in biosensing when nanostructured electrodes are used.

\section{Experimental Methods}

Nanopillar array electrodes (NAEs) with three different pillar heights were fabricated using a template method [12]. In fabricating these electrodes, a layer of gold film about $150 \mathrm{~nm}$ thick was first sputter-coated onto one side of a porous anodic alumina (PAA) circular disc $(\mathrm{d}=25$ $\mathrm{mm}$; Whatman Inc, Maidstone, England) having an average pore diameter of $150 \mathrm{~nm}$ using a SPI sputter coater (Structure probe Inc, West Chester, PA). Then, a thicker gold layer was electrodeposited on top of the sputtered gold film to form a strong supporting base in an Orotemp24 gold plating solution (Technic Inc, Cranston, Rhode Island) with a current density of $5 \mathrm{~mA} / \mathrm{cm}^{2}$ for two minutes. This supporting base was masked with Miccrostop solution (Pyramid plastics Inc., Hope, Arkansas) for insulation. After that, gold nanopillars were electrodeposited through the open pores of the PAA disc from the uncoated side under an electrical current density of 5 $\mathrm{mA} / \mathrm{cm}^{2}$ at $65^{\circ} \mathrm{C}$. The deposition time was varied for achieving nanopillars of different heights. For this study, specimens with three different nanopillar heights were prepared with the electrodeposition time controlled at 1 , 7 and 15 minutes. After nanopillar deposition, the PAA disc was dissolved in $2.0 \mathrm{M} \mathrm{NaOH}$ resulting in a thin gold sheet with arrays of vertically standing gold nanopillars. The fabricated specimens were cut into small square pieces (about $3.2 \times 3.2 \mathrm{~mm}^{2}$ ) and they were grouped into specimens A, B and C by their nanopillar height. For connecting the electrodes, a copper tape were attached to the backside of an electrode with the exposed part of the copper tape insulated using Miccrostop. Of these small specimens, some were used for scanning electron microscopy (SEM) imaging analyses, and some for electrochemical experiments (bare and functionalized conditions). For controls, two flat gold electrodes (one for bare and one for functionalized condition) with the same geometric size were prepared by depositing a thin film $(300 \mathrm{~nm})$ of gold on a titanium coated glass plate using a thermal evaporator (built in house). Prior to the electrochemical experiments, all electrodes (NAEs and flat) were cleaned by running cyclic voltammetry (CV) in $0.3 \mathrm{M} \mathrm{H}_{2} \mathrm{SO}_{4}$ between $-500 \mathrm{mV}$ and $1500 \mathrm{mV}$ until a stable $\mathrm{CV}$ curve was obtained for each specimen, and then washed with deionized water.

These electrodes were characterized in either bare or functionalized conditions. In the bare condition the cleaned electrodes were used directly, and in the functionalized condition the cleaned electrodes were further functionalized prior to use. To functionalize the electrodes, their surfaces were first modified with a SAM layer by placing them in a 75\% ethanol solution containing $10 \mathrm{mM}$ 3-mercaptopropionic acid. Then the SAM modified electrodes were rinsed in $75 \%$ ethanol and immersed in a $0.1 \mathrm{M} 2-(\mathrm{N}$ morpholino) ethanesulfonic buffer solution ( $\mathrm{pH}$ of 3.5 ) containing $2 \mathrm{mM}$ 1-ethyl-3-(3-dimethylaminopropyl) carbodiimide hydrochloride and $5 \mathrm{mM} \mathrm{N}$-hydroxysuccinimide for activation for two hours. After washing in phosphate buffer solution (PBS), the activated NAEs were placed in PBS solution at $\mathrm{pH} 7.4$ containing $1 \mathrm{mg} / \mathrm{ml}$ of glucose oxidase for two hours under constant stirring. The reason for setting the immobilization time to two hours is that according to literature [13], enzyme loading reaches its maximum in about 2 hours and it saturates afterwards. From the electrochemical experiments, the amperometric current responses of both bare and functionalized NAEs along with flat controls were measured using a conventional three-electrode cell with an $\mathrm{Ag} / \mathrm{AgCl}$ reference electrode and a platinum counter electrode with the Multistat 1480 (Solartron Analytical, Houston TX, USA) electrochemical system. 
For the bare-electrodes, their amperometric current responses at different concentrations of $\mathrm{K}_{4} \mathrm{Fe}(\mathrm{CN})_{6}$ to each incremental addition of $80 \mu \mathrm{l}$ of $1 \mathrm{M} \mathrm{K}_{4} \mathrm{Fe}(\mathrm{CN})_{6}$ to a $20 \mathrm{ml}$ solution containing $0.5 \mathrm{M} \mathrm{Na}_{2} \mathrm{SO}_{4}$ (equivalent to a $4 \mathrm{mM}$ increase in $\mathrm{K}_{4} \mathrm{Fe}(\mathrm{CN})_{6}$ concentration) were measured at a constant electrode potential of $350 \mathrm{mV}$ (vs. $\mathrm{Ag} / \mathrm{AgCl}$ ), and the change in the current response upon the change in $\mathrm{K}_{4} \mathrm{Fe}(\mathrm{CN})_{6}$ concentration for both the NEAs and flat electrode was determined. For the functionalized NAEs, the amperometric current responses to each incremental addition of $50 \mu \mathrm{l}$ of $1 \mathrm{M}$ glucose to a $20 \mathrm{ml}$ PBS solution (equivalent to a $2.5 \mathrm{mM}$ increase in glucose concentration) containing $3 \mathrm{mM}$ p-benzoquinone as a mediator were measured at a constant potential of $350 \mathrm{mV}$ (vs. Ag/ $\mathrm{AgCl})$. In all experiments, the background current of all electrodes was allowed to stabilize before drops of target species were added. Prior to these experiments the electrolyte solution was deaerated with nitrogen and during experiments the solution was blanketed with nitrogen and stirred constantly at $600 \mathrm{rpm}$.

For glucose detection, the electrode reactions in the present study can be described by the following cascading events [1]. With the catalysis of glucose oxidase (GOXFAD), glucose was first oxidized into gluconolactone with GOX-FADH $H_{2}$ as a by-product. The GOX-FADH ${ }_{2}$ was then converted back to its oxidized form (GOX-FAD) by the pbenzoquinone mediator in the solution. The mediator itself was converted back to its original form by oxidation at the electrode surface, through which free electrons were generated and picked up by the electrode to produce a current response. These cascading events can be expressed by the following reactions:

Glucose + GOX-FAD $\rightarrow$ Gluconolac tone + GOX-FADH $_{2}$

$$
\begin{gathered}
\mathrm{GOX}-\mathrm{FADH}_{2}+2 \text { Mediator }_{\mathrm{ox}} \rightarrow \\
\mathrm{GOX}-\mathrm{FAD}+2 \text { Mediator }_{\mathrm{red}}+2 \mathrm{H}^{+} \\
2 \text { Mediator }_{\mathrm{red}} \rightarrow 2 \text { Mediator }_{\mathrm{ox}}+2 \mathrm{e}^{-}
\end{gathered}
$$

Based on these enzymatic events and the current measurements from the functionalized electrodes, we performed an enzymatic-kinetics study to determine the enzyme activity in the functionalized electrodes by using the Michaelis-Menten equation:

$$
I_{s}=\frac{I_{\max } S}{K_{m}+S}
$$

Where $I_{s}$ is the steady-state current measured at each glucose concentration, $I_{\max }$ the maximum current attainable, $K_{m}$ the apparent Michaelis-Menten constant, and $S$ the concentration of the target species (i.e., glucose in this case). The parameter $K_{m}$ describes the enzymatic activity of glucose: the smaller the $K_{m}$ value is, the more efficient the enzymatic reaction is. When $K_{m}$ of an enzymatic biosensor is larger than the $K_{m}$ value of the freely dissolved enzyme, it usually implies that the enzyme immobilized in the biosensor is less efficient in oxidizing glucose than the dissolved enzyme [14]. In this study, the $K_{m}$ values for the functionalized electrodes were determined by performing nonlinear curve fit using Eq.4 to the measured current-concentration data.

\section{Simulation Procedures}

To elucidate the effects of reaction kinetics and mass transport on the current response of bare and functionalized NAEs, we simulated such an electrochemical process using a finite element analysis method with commercial software COMSOL Multiphysics (COMSOL Multiphysics, Burlington, MA). To simplify the situation we considered two dimensional situations. As shown schematically in Figure 1, a set of NAEs (with a width and a spacing of 200 $\mathrm{nm}$ for the pillars, and an overall dimension of $5 \mu \mathrm{m} \times 4.3$ $\mu \mathrm{m}$ for the electrode) was placed in a circular electrochemical cell containing a supporting electrolyte. In this simulation, a bare and a functionalized (with glucose oxidase) NAEs as well as a flat electrode with the same planar area (as a control) were considered.

For the electrode reaction at the functionalized NAEs, we assumed that glucose was consumed at a flux of $J_{g}$ at the electrode surface to produce the mediator in its reduced-

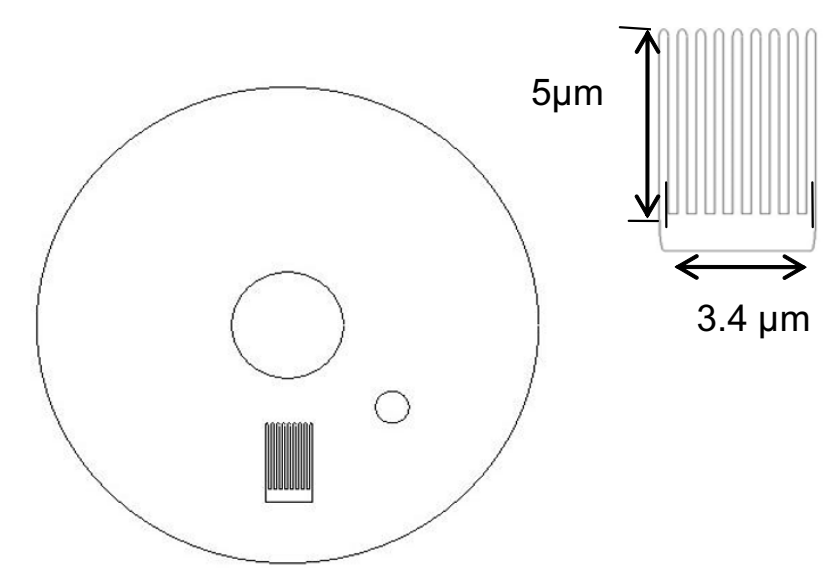

Figure I

A 2D model of a circular electrochemical cell containing a functionalized nanopillar electrode. The inner center circle is for generating the swirling vortex force to stir the solution and the small off-center circle is for creating a drop of uniform concentration of glucose prior to the kinetics analysis. A magnified view of the nanopillar electrode is shown at the upper right corner. 
form at a flux of $J_{M}$. Here $J_{g}$ and $J_{M}$ can be described by the following equations:

$$
\begin{gathered}
J_{\text {glucose }}=k c_{G} \\
J_{M}=k c_{G}-k_{0} c_{M} \exp \left(-\alpha F\left(E-E_{s t d}\right) / R T\right)
\end{gathered}
$$

where $k$ represents the rate constant for Eq. $1, c_{\mathrm{G}}$ the concentration of glucose, $c_{M}$ the concentration of mediator, $k_{0}$ the standard rate constant, $\alpha$ the charge transfer coefficient, $F$ the Faraday constant, $E$ the electrode potential, and $E_{s t d}$ the standard potential of the mediator. To simulate the actual event, the electrode was held at a constant overpotential of $350 \mathrm{mV}$. Under this condition, the reduced-form mediator was oxidized at the electrode surface to generate a current flux of $J_{c}$ :

$$
J_{C}=-2 k_{0} c_{M} \exp \left(-\alpha F\left(E-E_{s t d}\right) / R T\right)
$$

With these considerations, the amperometric current response of the electrodes in response to a drop of glucose was determined while the electrolyte solution was constantly stirred by a swirling vortex force applied at the center of the cell.

For the electrode reaction at the bare-electrode, we considered the redox of $\mathrm{K}_{4} \mathrm{Fe}(\mathrm{CN})_{6}$ with the reduction flux of $\mathrm{K}_{4} \mathrm{Fe}(\mathrm{CN})_{6}$ governed by:

$$
\begin{gathered}
J_{F}=-k_{0 F} c_{F 1} \exp \left(-\alpha F\left(E-E_{s t d}{ }^{\prime}\right) / R T\right)+ \\
k_{0 F} c_{F 2} \exp \left(-\alpha F\left(E-E_{s t d}\right) / R T\right)
\end{gathered}
$$

where $k_{0 F}$ is the electron transfer rate for both ferrocyanide and ferricyanide (assumed to be the same), $c_{F 1}$ the concentration of ferrocyanide, $c_{F 2}$ the concentration of ferricyanide, $E$ the electrode potential, and $E_{s t d}$ the standard potential of ferro- and ferri-cyanide.

Besides the reaction kinetics discussed above, the mass transport in these electrochemical processes was mainly governed by diffusion and convection for the mobile species such as glucose and $\mathrm{K}_{4} \mathrm{Fe}(\mathrm{CN})_{6}$. The electromigration was ignored because of the presence of the supporting electrolyte in a high concentration.

After these considerations, the diffusion/convection-controlled electrochemical reaction problems upon a step potential excitation $(350 \mathrm{mV})$ at the electrode were solved using the combined Electrokinetic-Flow and NavierStokes applications in COMSOL Multiphysics. In the simulation process, two initial analyses were performed. First, a stationary nonlinear analysis in Navier-Stokes mode was performed for reaching a fully developed vortex flow inside the center inner circle (see Figure 1), and then a stationary nonlinear analysis in Electrokinetic-Flow mode
Table I: Material constants and kinetic parameters used in the simulation [15]

\begin{tabular}{ccc}
\hline Parameter & & Value \\
\hline$k_{0}$ & Standard rate constant & $1.5 \times 10^{-3}(\mathrm{~m} / \mathrm{s})$ \\
$\varepsilon_{B}$ & Association factor & 2.6 \\
$\alpha$ & Charge transfer coefficient & 0.5 \\
$T$ & Absolute temperature & $298(\mathrm{~K})$ \\
$\mu$ & Viscosity & $1.1(\mathrm{cP})$ \\
$V_{A}$ & Molar volume & $0.1176\left(\mathrm{~m}^{3} / \mathrm{mol}\right)$ \\
$r_{p}$ & Pore radius & $200 \times 10^{-9}(\mathrm{~m})$ \\
$L$ & Pore length & $5 \times 10^{-6}(\mathrm{~m})$ \\
$k$ & Surface reaction rate & $5 \times 10^{-4}, 5 \times 10^{-5}, 5 \times 10^{-7}$ \\
& constant & $(\mathrm{m} / \mathrm{s})$ \\
$M_{B}$ & Molecular weight of water & 18 \\
$R$ & Gas constant & $8.31(\mathrm{~J} / \mathrm{K} . \mathrm{mol})$ \\
$F$ & Faraday constant & $9.648 \times 10^{4}(\mathrm{C} / \mathrm{mol})$ \\
$D_{F}$ & Diffusivity of ferro- and & $8 \times 10^{-10} \mathrm{~m}^{2} / \mathrm{s}$ \\
& ferri-cyanide &
\end{tabular}

was performed for producing a uniform initial concentration of glucose within the off-center inner circle (see Figure 1), much like dropping a small volume of glucose into the solution. After these initial steps, time dependent analyses were performed. For the kinetic constants, literature values [15] including the diffusivity of ferrocyanide and ferricyanide listed in Table 1 were used. The values for the diffusivity of glucose and the mediator, which are not readily available in the literature, were calculated using the following equation [16]:

$$
D_{A B}=\frac{1.17 \times 10^{-13}\left(\varepsilon_{B} M_{B}\right)^{\frac{1}{2}} T}{\mu V_{A}^{0.6}}
$$

where A represents the solute (e.g., glucose or the mediator) and $\mathrm{B}$ the solvent (e.g., water), $\varepsilon_{B}$ the association factor of the solvent, $M_{B}$ the molecular weight of the solvent, $\mu$ the viscosity of solution, $V_{A}$ the molar volume of solute glucose, and $T$ the absolute temperature.

\section{Results and Discussion}

Figure 2 shows three SEM images of electrodes Nano A, B and $\mathrm{C}$. In all specimens, the nanopillars had a diameter of about $150 \mathrm{~nm}$. From the side views (see insets in Fig. 2) of the specimens, the height of the nanopillars is estimated to be $1 \mu \mathrm{m}, 2.5 \mu \mathrm{m}$ and $6 \mu \mathrm{m}$ for specimen $\mathrm{A}, \mathrm{B}$ and $\mathrm{C}$, respectively. From these images, we noted that in electrodes with taller nanopillars (e.g., Nano B and Nano C) there are slight bunching deformations in nanopillars. This kind of deformation is caused by the capillary interaction (during the wetting of the electrodes) compounded by the reduced flexure rigidity of these taller nanopillars [12]. Also shown here is the cyclic voltammograms for 


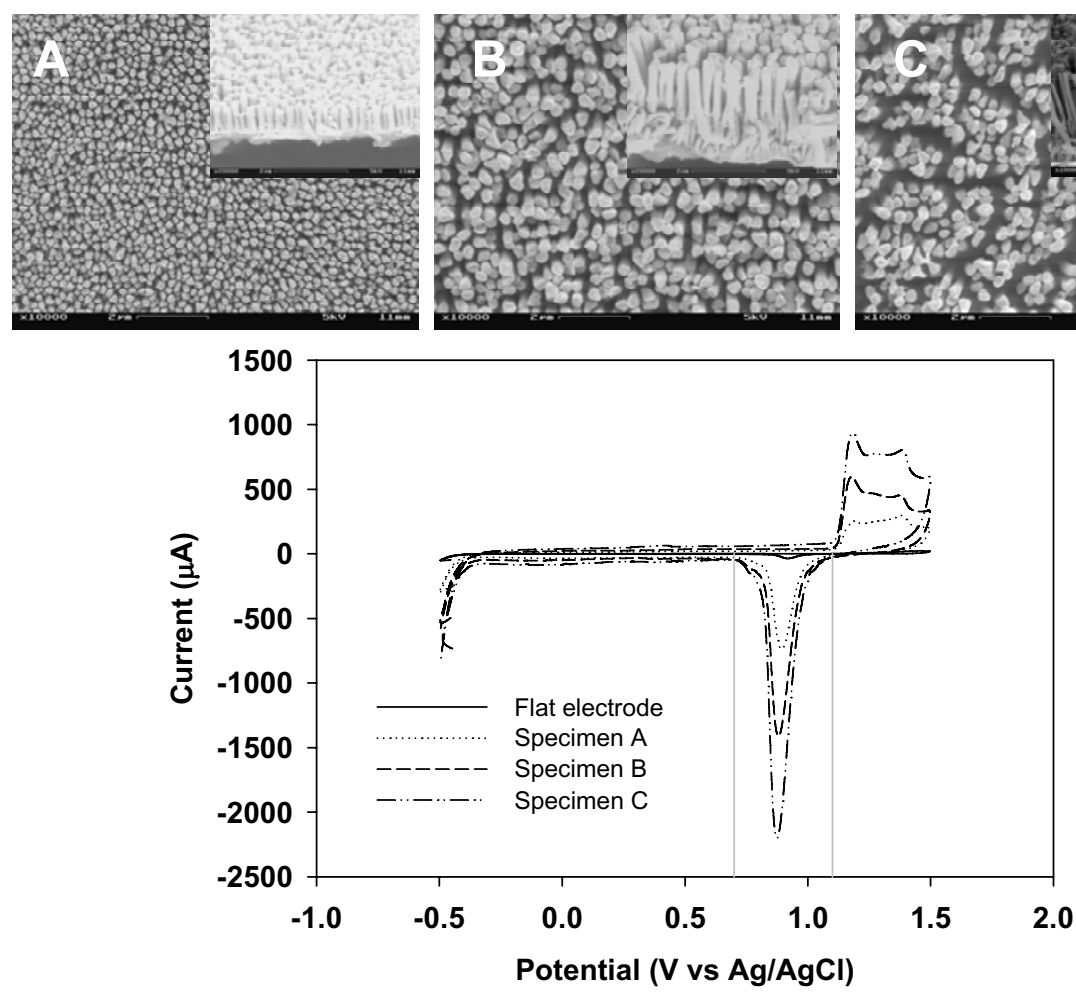

Figure 2

SEM images of Nano A, B and C specimens with insets showing a side-view of the specimens. Also shown here are the cyclic voltammograms obtained for three bare NAEs and a flat electrode.

three NAEs and a flat electrode measured in $0.3 \mathrm{M}$ sulphuric acid. In all these voltammograms, a reduction peak is seen in between $0.70 \mathrm{~V}$ and $1.1 \mathrm{~V}$. To quantify the difference in the height of the nanopillars in these NAEs, we defined a roughness ratio as the area under the reduction peak (calculated by integrating the voltammogram from $0.70 \mathrm{~V}$ to $1.1 \mathrm{~V}$ ) of a NAEs electrode divided by that of the flat electrode. The roughness ratio was found to be about 20, 38.8 and 63.4 for specimens A, B and C, respectively (see Table 2).
Figure 3A shows the amperometric current response for the bare electrodes (NAEs and flat) at various $\mathrm{K}_{4} \mathrm{Fe}(\mathrm{CN})_{6}$ concentrations. In general, all the NAEs exhibited a higher current than the flat electrode at each $\mathrm{K}_{4} \mathrm{Fe}(\mathrm{CN})_{6}$ concentration. To further quantify the sensing performance of these bare electrodes, we analyzed the relationship between the current response and $\mathrm{K}_{4} \mathrm{Fe}(\mathrm{CN})_{6}$ concentration by a linear regression analysis. Figure $3 \mathrm{~B}$ shows the variation of the steady-state amperometric current with the concentration of $\mathrm{K}_{4} \mathrm{Fe}(\mathrm{CN})_{6}$ (from $4 \mathrm{mM}$ to $24 \mathrm{mM}$ )

Table 2: The roughness ratio, detection sensitivity, $I_{\max }$ and $K_{m}$ obtained from experiments

\begin{tabular}{cccccc}
\hline Specimen & Roughness Ratio & $\begin{array}{c}\text { Sensitivity of bare electrodes } \\
\text { to } \mathrm{K}_{4} \mathrm{Fe}(\mathrm{CN})_{6}\left(\mu \mathrm{A} \cdot \mathrm{mMM}^{-1} \cdot \mathrm{cm}^{-2}\right)\end{array}$ & $\begin{array}{c}\text { Sensitivity of } \\
\text { functionalize } \\
\text { electrodes to glucose } \\
\left(\mu \mathrm{A} \cdot \mathrm{mM}^{-1} \cdot \mathrm{cm}^{-2}\right)\end{array}$ & $I_{\max }$ glucose $(\mu \mathrm{A})$ & $K_{m} g$ lucose $(\mathrm{mM})$ \\
\hline Flat & 1.0 & 19.30 & 0.27 & 1.34 & 24.8 \\
Nano A & 20.0 & 41.40 & 0.91 & 5.06 & 29.3 \\
Nano B & 38.8 & 41.05 & 1.80 & 2.1 & 32.6 \\
Nano C & 63.4 & 41.70 & 3.13 & 23.0 & 52.0 \\
\hline
\end{tabular}



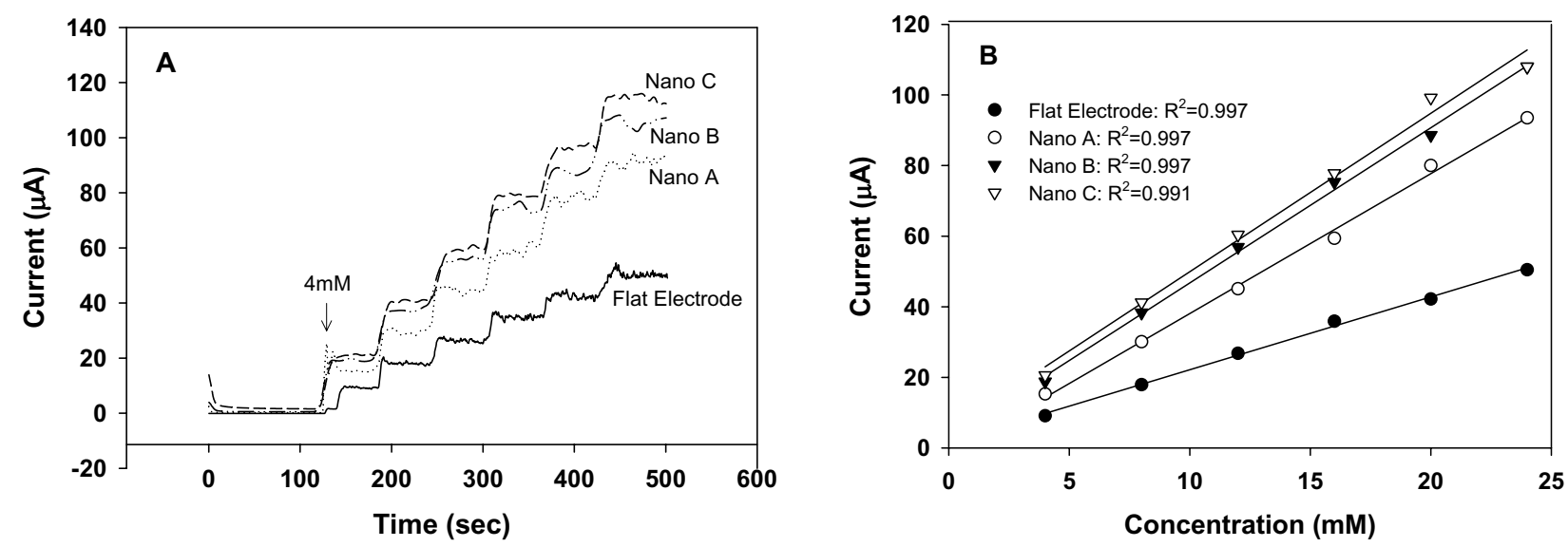

\section{Figure 3}

(A) Amperometric current responses obtained for the bare NAEs and flat electrode when incremental drops of $\mathrm{K}_{4} \mathrm{Fe}(\mathrm{CN})_{6}$ were added to the solution. (B) Calibration curves obtained based on a linear-regression analysis for the relationship between the steady-state current and $\mathrm{K}_{4} \mathrm{Fe}(\mathrm{CN})_{6}$ concentration.

along with the corresponding regression lines. By taking the slope of the regression lines and normalizing it with respect to the geometrical area of the electrodes $(3.2 \mathrm{~mm}$ $\times 3.2 \mathrm{~mm}$ ), we obtained sensitivity values for the electrodes and these values were listed in Table 2. Clearly, for all the bare electrodes, the NAEs showed sensitivity about two times higher than that of the flat electrode.

One surprising observation, however, was that the sensitivity of these bare NAEs did not increase with the increase of the roughness ratio. This implies that the benefit of the increased surface area due to nanopillars has not been fully realized. It seems that only the top part of the nanopillars has contributed to the increase of active electrode surface for electron transfer, which may explain why there is only a two-fold increase in the current responses of all the NAEs as compared with the flat electrode. We speculate that the electroactive species $\mathrm{K}_{4} \mathrm{Fe}(\mathrm{CN})_{6}$ may encounter certain difficulties in its transport to the small spaces between the bare nanopillars as the result of either a low diffusivity or a fast electron transfer rate constant. With a low diffusivity, it would be difficult for $\mathrm{K}_{4} \mathrm{Fe}(\mathrm{CN})_{6}$ to diffuse deep into the small spaces between the nanopillars, while with a fast electron transfer rate constant, most of the species $\mathrm{K}_{4} \mathrm{Fe}(\mathrm{CN})_{6}$ would get oxidized near the top ends of the nanopillars before it diffuses down the gaps. Under such a circumstance, it is conceivable that only the top regions of the nanopillars are serving their active duty in transferring electrons.

Figure 4A shows the amperometric currents for the functionalized NAEs and flat electrode at various glucose concentrations. Again, all the NAEs exhibited a higher current response than the flat electrode at each glucose concentra- tion. Note that in each incremental step, the current response of Nano $\mathrm{C}$ is still rising indicating that it has not reached its steady state. We believe this phenomenon is due to the increased response times for electrodes with taller nanopillars. However, for a quick comparison between these nano electrodes, we took a more conservative approach to get the current readings for Nano $\mathrm{C}$ at the same time as for Nano B and Nano C.

Figure 4B shows the variations of the steady-state amperometric current with glucose concentration (from $2.5 \mathrm{mM}$ to $15 \mathrm{mM}$ ) along with the corresponding linear regression lines. By taking the slope of the regression lines and normalizing it with respect to the geometric area of the electrode in each case, we obtained the sensitivity measurement for the functionalized electrodes (NAEs and flat). From the obtained sensitivity values listed in Table 2, we observed that unlike in the bare electrode cases, the sensitivity of NAEs increases as the roughness ratio increases. The highest sensitivity value (Nano $\mathrm{C}$ ) is about $3.13 \mu \mathrm{A} \cdot \mathrm{mM}^{-1} \cdot \mathrm{cm}^{-2}$ (about 12 times higher than that for a flat electrode) which is significantly higher than the value reported for a gold nanotube electrode (0.4 $\mu \mathrm{A} \cdot \mathrm{mM}^{-1} \cdot \mathrm{cm}^{-2}$ ) [8]. So for the functionalized NAEs, increasing the surface roughness of the NAEs does contribute to an increase in detection sensitivity.

Figure 5 shows the variations of the steady-state amperometric current with the glucose concentration over a wider concentration range ( $2.5 \mathrm{mM}$ to $30 \mathrm{mM}$ ). By performing nonlinear curve fitting to the data using Eq.4, we obtained values for $K_{m}$ and $I_{\max }$ in each case as listed in Table 2 . Clearly, both $I_{\max }$ and $K_{m}$ values are higher for the NAEs than for the flat electrode and they increase as the rough- 

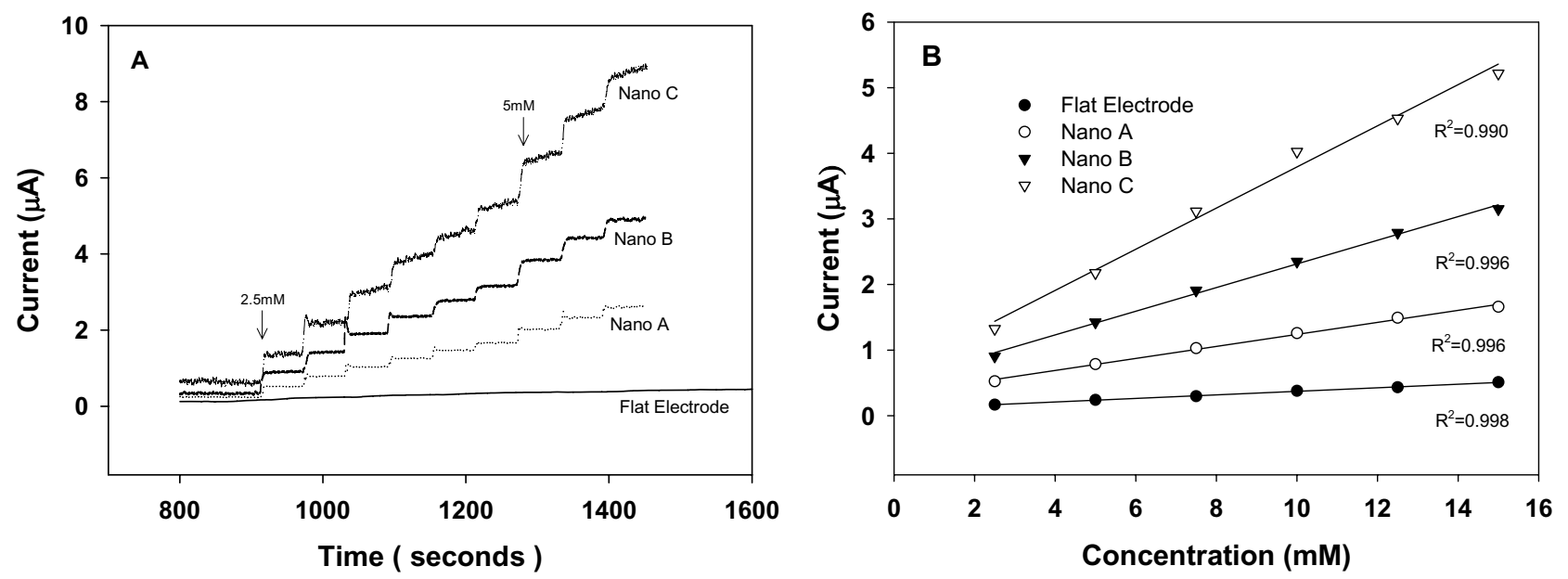

\section{Figure 4}

(A) Amperometric current responses obtained for the functionalized NAEs and flat electrode when incremental drops of glucose were added to the solution. (B) Calibration curve obtained based on a linear-regression analysis for the relationship between the steady-state current and glucose concentration (from $2.5 \mathrm{mM}$ to $15 \mathrm{mM}$ ).

ness ratio increases. Furthermore, the $K_{m}$ values for all the NAEs are larger than the reported intrinsic $K_{m}$ value of 25 $\mathrm{mM}$ for dissolved glucose oxidase [17]. This indicates that the activity of the enzyme immobilized on these NAEs has actually been lowered as compared with the freely dissolved enzyme, which further suggests that the increase in sensitivity in the functionalized NAEs is due to factors other than enzyme activity.

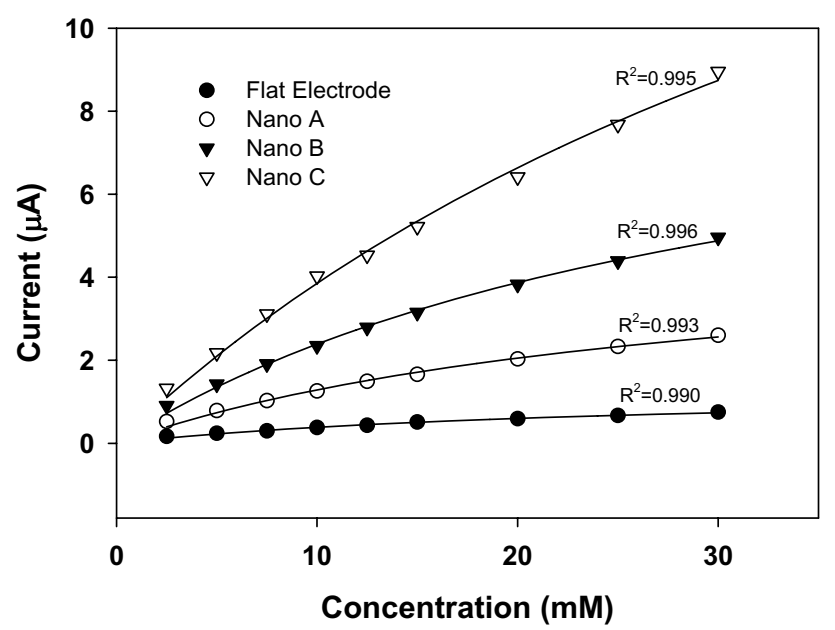

\section{Figure 5}

Variation of the steady-state current with glucose concentration (from $2.5 \mathrm{mM}$ to $30 \mathrm{mM}$ ) for various functionalized electrodes along with the nonlinear-fitted curves based on the Michaelis-Menten equation.
In comparing the bare with the functionalized electrodes, we found that the highest nanostructure-induced sensitivity increase for the functionalized electrodes (12 times) is higher than that for the bare electrodes (2 times). This could be due to the difference in electrochemical species involved (i.e., glucose versus $\mathrm{K}_{4} \mathrm{Fe}(\mathrm{CN})_{6}$ ). These two electroactive species, however, have a similar diffusivity value $\left(8 \times 10^{-10} \mathrm{~m}^{2} / \mathrm{s}\right.$ for $\mathrm{K}_{4} \mathrm{Fe}(\mathrm{CN})_{6}$ and $7.6 \times 10^{-10} \mathrm{~m}^{2} / \mathrm{s}$ for glucose) [15]. This fact suggests that the difference in the reaction rate constant at the bare and functionalized electrodes may play a more dominate role in affecting the current response. It is also possible that such an increase in the sensitivity of functionalized NAEs is the result of heightened retention of the mediator during glucose detection [11].

To see the influence of the reaction rate constant on the current response of the NAEs, we now turn to the simulation results. Figure $6 \mathrm{~A}$ shows the simulated amperometric current obtained for a functionalized NAEs and a flat electrode in response to glucose at two different reaction rate constants: $1.5 \times 10^{-5}$ and $1.5 \times 10^{-7}(\mathrm{~m} / \mathrm{s})$. As expected, a higher current response was found for the nanopillar electrode than for the flat electrode (see Table 3). But the nanostructure-induced increase in the current response was affected significantly by the reaction rate constant of glucose. At a rate constant of $1.5 \times 10^{-5} \mathrm{~m} / \mathrm{s}$ the increase in current due to nanopillars was only 3.26 fold, whereas at a rate constant of $1.5 \times 10^{-7} \mathrm{~m} / \mathrm{s}$ the increase was 22.26 fold. This fact suggests that at a lower reaction rate constant more glucose will be able to diffuse into the deep space between the nanopillars to get oxidized, thus lead- 

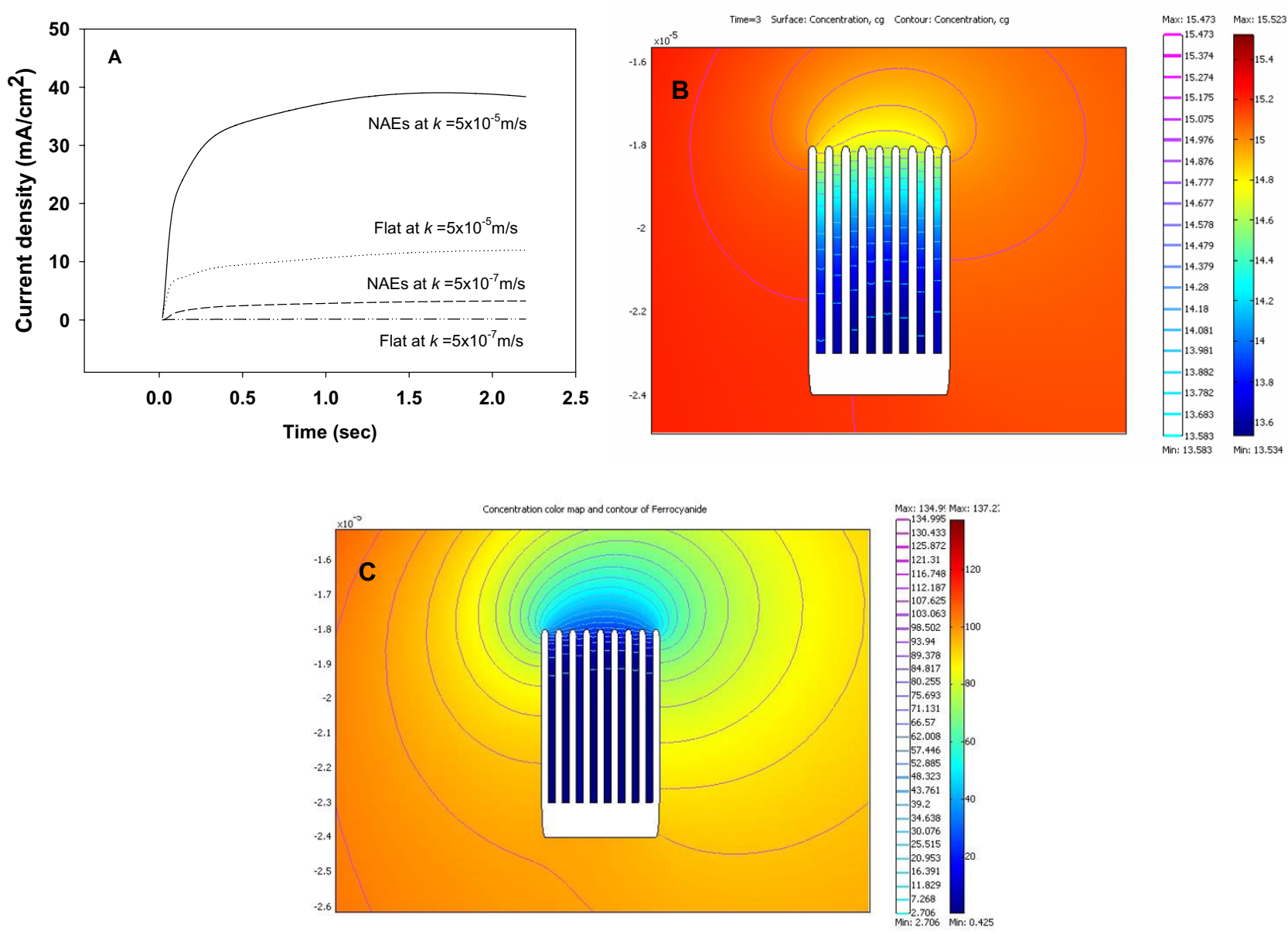

\section{Figure 6}

(A) Simulated current responses for a functionalized NAEs electrode and a flat electrode at a reaction rate constant of $5 \times 10$ $5 \mathrm{~m} / \mathrm{s}$ and $5 \times 10^{-7} \mathrm{~m} / \mathrm{s}$. (B) Contour plot for glucose concentration near the electrode at a reaction rate constant of $5 \times 10^{-7} \mathrm{~m} /$ s. (C) Contour plot for $\mathrm{K}_{4} \mathrm{Fe}(\mathrm{CN})_{6}$ concentration near the electrode at a reaction rate constant of $5 \times 10^{-4} \mathrm{~m} / \mathrm{s}$.

ing to a higher current response. By contrast, $\mathrm{K}_{4} \mathrm{Fe}(\mathrm{CN})_{6}$ has a rate constant of $1.5 \times 10^{-4}$, and at this rate constant the nanostructure-induced increase in current response is found to be only 1.28 fold (see Table 3 ). This is so because

Table 3: Steady-state amperometric current density obtained at different rate constants from computer simulation

\begin{tabular}{cccc}
\hline $\begin{array}{c}\text { Reaction rate } \\
\text { constant }(\mathrm{m} / \mathrm{s})\end{array}$ & \multicolumn{3}{c}{ Current Density $\left(\mathrm{mA} \cdot \mathrm{cm}^{-2}\right)$} \\
\cline { 2 - 4 } & Nano & Flat & Nano/Flat \\
\hline $\begin{array}{l}5 \times 10^{-4} \text { for } \\
\mathrm{K}_{4} \mathrm{Fe}(\mathrm{CN})_{6}\end{array}$ & 279.51 & 219.18 & 1.28 \\
$5 \times 10^{-5}$ for \\
$\begin{array}{c}\text { glucose } \\
5 \times 10^{-7} \text { for } \\
\text { glucose }\end{array}$ & 39.1 & 12.0 & 3.26 \\
\hline
\end{tabular}

at such a high reaction rate constant, $\mathrm{K}_{4} \mathrm{Fe}(\mathrm{CN})_{6}$ will get oxidized quickly at the top regions of the nanopillars before it can diffuse down to the space between the nanopillars. These arguments were supported by the fact that a higher glucose concentration was found at the bottom of the spaces between nanopillars in the case with a lower reaction rate constant: a concentration of $0.497 \mathrm{~mol} / \mathrm{m}^{3}$ and $13.583 \mathrm{~mol} / \mathrm{m}^{3}$ was found at the bottom of the spaces between nanopillars when the rate constant is $1.5 \times 10^{-5}$ $\mathrm{m} / \mathrm{s}$ and $1.5 \times 10^{-7} \mathrm{~m} / \mathrm{s}$, respectively. Figure $6 \mathrm{~B}$ shows a contour plot for glucose concentration at a rate constant of $1.5 \times 10^{-7} \mathrm{~m} / \mathrm{s}$, where it is seen that a significant amount of glucose reached to the bottom of the spaces between nanopillars. In the case of $\mathrm{K}_{4} \mathrm{Fe}(\mathrm{CN})_{6}$ its concentration is found to be zero at the bottom of the spaces between nanopillars (see Figure 6C). 
The results obtained from simulation are consistent with the prediction for the catalytic reactions in porous media based on the effectiveness factor $(\eta)$ and thiele modulus $(\phi)$ concepts. The value of $\eta$ can be determined by using the following formula [18]:

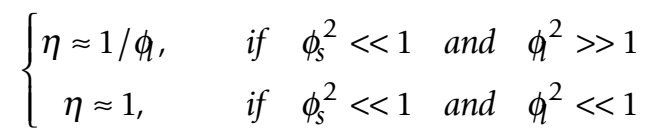

Here $\phi_{s}^{2}$ and $\phi^{2}$ are the thiele moduli calculated based on the transverse and longitudinal diffusion times, and they are defined by $\phi_{s}^{2}=2 k r_{p} / D_{G^{\prime}} \phi^{2}=2 k L^{2} / r_{p} D_{G^{\prime}}$ where $r_{p}$ is the pore radius, $L$ is the pore length, $D_{G}$ is the glucose diffusivity and $k$ is the surface rate constant. For a surface rate constant of $5 \times 10^{-4} \mathrm{~m} / \mathrm{s}$, we calculated $\phi_{s}^{2}=0.131$ and $\phi^{2}=328.8$. Under this condition, $\eta$ is found to be $\eta$ $\approx 1 / \varphi=0.055$. This low effectiveness factor will hinder the transport of the target species to the spaces between the nanopillars. For a lower surface reaction rate constant of 5 $\times 10^{-7} \mathrm{~m} / \mathrm{s}$, we calculated $\phi_{s}^{2}=131.5 \times 10^{-6}$ and $\phi^{2}=$ 0.328 , and under this condition $\eta$ will be close to unity ( $\eta$ $\approx 1$ ). This high effectiveness factor will surely enable more efficient transport of glucose to the functionalized surfaces in between the nanopillars.

The above results clearly indicate that the enhanced current response in glucose sensing with functionalized NAEs can be attributed to the effective mass transport facilitated by the relatively lower reaction rate constant of glucose. This fact suggests that to reap the true benefit of using nanostructured electrodes for enhancing the performance of biosensors, it is necessary to optimize the geometry of the nanopillars (their diameter, spacing and height) in order to accommodate the specific analyte species in terms of its reaction kinetics and mass transport.

\section{Conclusion}

The role of reaction kinetics and mass transport in biosensing using electrodes integrated with nanopillars of different heights was investigated. In an electrochemical based detection, the increased active surface area due to the addition of nanopillars may lead to enhanced sensing performances only when the reaction rate constant of the target species is low. At a higher reaction rate constant, only the top part of the nanopillar modified electrodes will serve the purpose for transferring electrons. To reap the benefit of using nanostructured electrodes for improving the sensing performances, it is necessary to optimize the geometry of the nanopillars to accommodate the specific analyte species in terms of its reaction kinetics and mass transport.

\section{Competing interests}

The author(s) declare that they have no competing interests.

\section{Authors' contributions}

VA, XY, EK and YLR contributed to the design of the study, the acquisition and analysis of data, and the writing of the manuscript. GZ contributed to the design and coordination of the study and participated in the writing of the manuscript. All authors read and approved the final manuscript.

\section{Acknowledgements}

This work was partially supported by the National Science Foundation (ECS-0304340), the Faculty of Engineering and the College of Agricultural and Environmental Science at the University of Georgia.

\section{References}

I. Berchmans S, Sathyajith R, Yegnaraman V: Layer-by-layer assembly of I,4-diaminoanthraquinone and glucose oxidase. Materials Chemistry and Physics 2003, 77:390-396.

2. Gooding JJ, Erokhin P, Hibbert DB: Parameters important in tuning the response of monolayer enzyme electrodes fabricated using self-assembled monolayers of alkanethiols. Biosensors \& Bioelectronics 2000, I 5:229-239.

3. Gooding JJ, Praig VG, Hall EAH: Platinum-catalyzed enzyme electrodes immobilized on gold using self-assembled layers. Analytical Chemistry 1998, 70:2396-2402.

4. Losic D, Gooding JJ, Shapter JG, Hibbert DB, Short K: The influence of the underlying gold substrate on glucose oxidase electrodes fabricated using self-assembled monolayers. Electroanalysis 2001, I 3: I 385-1393.

5. Gao M, Gordon LD: Biosensors Based on Aligned Carbon Nanotubes Coated with Inherently Conducting Polymers. Electroanalysis 2001, I5:1089-1094.

6. Uang YM, Chow TC: Criteria for Designing a Polypyrrole Glucose Biosensor by Galvanostatic Electropolymerization. Electroanalysis 2002, I4: I564-I570.

7. Qiaocui S, Tuzhi P, Yunu Z, Yang CF: An Electrochemical Biosensor with Cholesterol Oxidase/Sol-Gel Film on a Nanoplatinum/Carbon Nanotube Electrode. Electroanalysis 2005, | 7:857-86|.

8. Delvaux M, Demoustier-Champagne S: Immobilisation of glucose oxidase within metallic nanotubes arrays for application to enzyme biosensors. Biosensors \& Bioelectronics 2003, I 8:943-95I.

9. Wang J, Musameh M: Carbon Nanotube/Teflon Composite Electrochemical Sensors and Biosensors. Anal Chem 2003, 75:2075-2079.

10. Bharathi S, Nogami M: A glucose biosensor based on electrodeposited biocomposites of gold nanoparticles and glucose oxidase enzyme. Analyst 2001, I 26:1919-1922.

II. Wang J, Nosang M, Minhee Y, Harold M: Glucose oxidase entrapped in polypyrrole on high-surface-area $\mathrm{Pt}$ electrodes: a model platform for sensitive electroenzymatic biosensors. Journal of Electroanalytical Chemistry 2005, 575:139-146.

12. Anandan V, Rao YL, Zhang G: Nanopillar array structures for high performance electrochemical sensing. International journal of nanomedicine 2006, I:73-79.

13. Gooding JJ, Erokhin P, Losic D, Yang W, Policarpio V, Liu J, Ho F, Situmorang M, Hibbert DB, Shapter JG: Parameters important in fabricating enzyme electrodes using self-assembled monolayers of alkanethiols. Anal Sci 200I, I 7(I):3-9.

14. Harper AC: Modified electrodes for amperometric determination of glucose and glutamate using mediated electron 
transport. In PhD Thesis Virginia Polytechnic Institute and State University, Chemistry Department; 2005.

15. Winkler K: The kinetics of electron transfer in $\mathrm{Fe}(\mathrm{CN})_{6}^{4-13-}$ redox system on platinum standard-size and ultramicroelectrodes. Journal of Electroanalytical Chemistry 1995, 388:15I-I59.

16. Heinsohn RJ, Cimbala JM: Indoor Air Quality Engineering: Environmental Health and Control of Indoor Pollutants. New York: Marcel Dekker Inc; 2003.

17. Calvo EJ, Wolosiuk A: Supramolecular Architectures of Electrostatic Self-Assembled Glucose Oxidase Enzyme Electrodes. ChemPhysChem 2004, 5:235-239.

18. Balakotaiah $V$, Gupta N: Controlling regimes for surface reactions in catalyst pores. Chemical Engineering Science 2000, 55:3505-35I4

Publish with Bio Med Central and every scientist can read your work free of charge

"BioMed Central will be the most significant development for disseminating the results of biomedical research in our lifetime. "

Sir Paul Nurse, Cancer Research UK

Your research papers will be:

- available free of charge to the entire biomedical community

- peer reviewed and published immediately upon acceptance

- cited in PubMed and archived on PubMed Central

- yours - you keep the copyright

Submit your manuscript here:

http://www.biomedcentral.com/info/publishing_adv.asp
BioMedcentral 